\title{
Investigation of dynamic of unconsolidated materials using two-color digital holography
}

\author{
P. Tankam ${ }^{1, \mathrm{a}}$, P. Picart ${ }^{1,2}$, D. Mounier ${ }^{2,3}$, J.-P. Boileau ${ }^{2}$, V. Tournat ${ }^{1}$, and V. Gusev ${ }^{1,3}$ \\ ${ }^{1}$ LAUM CNRS, Université du Maine, Avenue Olivier Messiaen, 72085 LE MANS Cedex 9, France \\ ${ }^{2}$ École Nationale Supérieure d'Ingénieurs du Mans, rue Aristote, 72085 Le Mans Cedex 9, France \\ ${ }^{3}$ LPEC CNRS, Université du Maine, Avenue Olivier Messiaen, 72085 LE MANS Cedex 9, France
}

\begin{abstract}
The paper presents a two-color digital holographic interferometer. The set-up is devoted to the study of the fundamental dynamic of unconsolidated materials. Optical configuration and algorithms to recover the optical phase of two-color digitally encoded holograms are described. The method is based on a spatial-color-multiplexing scheme in which holographic reconstruction is performed using adapted wavelength zero-padding and reconstructing distance. Experimental results are presented in the case of granular media excited in the frequency range $400 \mathrm{~Hz}-3000 \mathrm{~Hz}$ and exhibits the 3D movement.
\end{abstract}

\section{Introduction}

Digital holography became properly available since its confirmation was established in 1994 [1]. In few years, a lot of spectacular applications have been demonstrated such as microscopic imaging and phase-contrast digital holographic microscopy [2], surface shape measurement and contouring and also material property investigations [3], vibrations analysis with pulsed lasers and time averaging, also multidimensional dynamic investigations [4]. The perspective of measurement of 3D vibration motions using digital Fresnel holography could be very important for getting insight in the fundamental dynamics of disordered granular materials. It is established that continuum elasticity, applied to assemblage of grains, actually decreases significantly below a length scale of typically 30 to 50 grain sizes [5]. At shorter scales the response of an unconsolidated granular material even to normal compression loading is nonaffine, i.e. contains additional component of the displacement field of predominantly rotational nature, which is rather controlled not by the symmetry of loading but by the characteristic of the disorder. Consequently 3D monitoring of the dynamics of disordered granular media could provide opportunity to study 3D nonaffine motion, to characterize its spatial scale and statistic (correlation) properties, and to attack the fundamental question [5]: "What modes of vibration contribute to vibration anomaly (Boson peak) in disordered materials?". In this paper, we present an experimental investigation of the 3D motion of granular medium by using a two-color digital holographic interferometer.

\section{Basics of digital color holographic reconstruction}

\footnotetext{
a e-mail : patrice.tankam.etu@univ-lemans.fr
}

This is an Open Access article distributed under the terms of the Creative Commons Attribution-Noncommercial License 3.0, which permits unrestricted use, distribution, and reproduction in any noncommercial medium, provided the original work is properly cited. 
In digital holography, numerical reconstruction of digitally encoded holograms is performed by use of a discrete version of the Fresnel transform associated with a zero-padding. However, a simultaneous multi-dimensional measurement will be possible only if it is possible to perfectly separate the contributions of each component of a 3D movement. For this, the size of each hologram along each wavelength must be quite similar during the reconstruction process in order to get a perfect pixel to pixel superimposition. Several algorithms have been developed in this sense [6,7]. However, algorithm presented in [6] needs a very long computation time and is not adapted to object which size is greater than $10 \mathrm{~mm}$. The computation of holograms along several wavelength needs to respect this fundamental property: the size of the object must be conserved and the number of sampling data point must be independent of the wavelength. The sampling pitches of digital holograms computed from the Fresnel transform are given along $\{x, y\}$ directions by $\Delta \eta=\lambda d_{0} / K p_{x}$ and $\Delta \xi=\lambda d_{0} / K p_{y}$. It is clear that if the wavelength changes, then the sampling pitch also changes. In order to simply our purpose, in all what follow we consider the problem along the $x$ direction.

Consider the case of two-wavelength holography with a red $(R)$ HeNe laser $\left(\lambda_{R}=632.8 \mathrm{~nm}\right)$ and a green $(G)$ DPSS laser $\left(\lambda_{G}=532 \mathrm{~nm}\right)$. Along each wavelength, we have sampling pitches given by $\Delta \eta_{R}=\lambda_{R} d_{0} / K_{R} p_{x}$ and $\Delta \eta_{G}=\lambda_{G} d_{0} / K_{G} p_{x}$ where $K_{R}$ and $K_{G}$ are the number of data points used in the discrete Fresnel transform. The main constraint on $K_{R}$ and $K_{G}$ is that they must be integer number, and furthermore we will chose them even. Then we must have $\Delta \eta_{j}=$ const for all color $j$. In the case of interest, this leads to a non integer ratio $K_{R} / K_{G}=\lambda_{R} / \lambda_{G}=1.189473$ leading to non integer value of $K_{R}$ relatively to $K_{G}$. In order to jump this point and avoid rounding on number of data points, one can chose $1000 K_{R} / K_{G}=$ integer, where integer $=1189$ or integer $=1190$, for the considered wavelengths. However, this approximation cannot lead exactly to $\Delta \eta_{G}=\Delta \eta_{R}$. To get the equality at a given precision, one can consider that the reconstructing distance is slightly different from that of the recording and that it depends on the wavelength. Thus, the sampling pitches in the image are now $\Delta \eta_{R}=\lambda_{R} d_{R} / K_{R} p_{x}$ and $\Delta \eta_{G}=\lambda_{G} d_{G} / K_{G} p_{x}$ (a similar relation holds for the $y$ direction), where $d_{R}$ and $d_{G}$ are respectively the reconstructing distance for the $R$ and $G$ holograms. To get exactly $\Delta \eta_{G}=\Delta \eta_{R}$ one can chose $d_{G}=d_{0}$ and $d_{R}$ to be proportional to $d_{G}$, such that $d_{R}=1189 / 1000 \times \lambda_{G} / \lambda_{R} \times d_{0}$ at a given precision. Symmetrically, one can consider ratio $1000 K_{G} / K_{R}=$ integer for which $\lambda_{G} / \lambda_{R}=0.840707$, integer $=840$ or integer $=841$ and one must chose $d_{G}=$ integer $/ 1000 \times \lambda_{R} / \lambda_{G} \times d_{R}$ at the wanted accuracy. Choosing $d_{R}=d_{0}$ leads to $d_{G}=$ integer $/ 1000 \times \lambda_{R} / \lambda_{G} \times d_{0}$. Note that there are few couples of values $\left\{K_{R}, K_{G}\right\}$ which respect the conditions "integers and even numbers". It is found that couple, for $1000 K_{G} / K_{R}=1189$ is $\left\{K_{G}, K_{R}\right\}=\{2000,2378\}$ (1), for $1000 K_{G} / K_{R}=1190$ is $\left\{K_{G}, K_{R}\right\}=\{2000,2380\}$ (2), for $1000 K_{R} / K_{G}=840$ is $\left\{K_{G}, K_{R}\right\}=\{2000,1680\}$ (3) and for $1000 K_{R} / K_{G}=841$ is $\left\{K_{G}, K_{R}\right\}=\{2000,1682\}$ (4).

One may wonder what is the best choice for the combination of the parameters and what consequences this may have on the reconstructing process. One clue can be found in [8]. It is shown that the change in the reconstruction distance introduces a focussing error in the image and that the enlarging of the impulse response of the process is given in the $x$ direction by:

$$
\rho_{x}^{d_{j}}=\left|N p_{x}\left(1+\frac{d_{j}}{d_{0}}\right)\right|
$$

$N$ being the effective pixel number of the recording sensor (a similar relation holds for $y$ ). In the following experiments, the sensor is a Pixel Fly CCD recording area including $(M \times N)=(1024 \times 1360)$ rows by columns with $p_{x}=p_{y}=4.65 \mu \mathrm{m}$. So consider that the object under interest is placed at $d_{0}=1400 \mathrm{~mm}$ from the recording area, with $N=1360$ and $p_{x}=4.65 \mu \mathrm{m}$, we have for configuration (1) $\rho_{x}^{d_{R}}=1360 \times 4.65|1-1499.40265 / 1500|=2.518 \mu \mathrm{m}$, for (2) it gives $\rho_{x}^{d_{R}}=2.798 \mu m$, for (3) it was computed $\rho_{x}^{d_{G}}=5.325 \mu \mathrm{m}$ and for (4) one gets $\rho_{x}^{d_{G}}=2.196 \mu \mathrm{m}$. These values can be compared with the theoretical spatial resolution given by $\rho_{x}^{R}=\lambda_{R} d_{0} / N p_{x}$ and $\rho_{x}^{G}=\lambda_{G} d_{0} / N p_{x}$ by calculating the 
ratio $\rho_{x}^{d_{G}} / \rho_{x}^{G}$ and $\rho_{x}^{d_{R}} / \rho_{x}^{R}$, giving $\rho_{x}^{d_{R}} / \rho_{x}^{R}=1.68 \%$ and $\rho_{x}^{d_{R}} / \rho_{x}^{R}=1.86 \%$ respectively for (1) and (2) and $\rho_{x}^{d_{G}} / \rho_{x}^{G}=4.22 \%$ and $\rho_{x}^{d_{G}} / \rho_{x}^{G}=1.74 \%$ for (3) and (4). This allows us to choose the best configuration that does not lead to much to enlarging of the spatial resolution in the reconstructed field. According to these numerical results, the best configuration for the reconstruction parameters is given by (1). This means that there are one pair of values $\left\{K_{G}, K_{R}\right\}=\{2000,2378\}$ which is optimal.

\section{Experimental set-up}

The set-up for studying dynamic of unconsolidated materials using two-color digital holography is composed of two cw lasers $(R, \mathrm{HeNe})$ and $(G$, DPSS) with different angles of incidence in order to be sensitive to the plane and out-of-plane vibration of the material (Fig. 1). A Mach-Zehnder interferometer produces the mixing between the two duplets of colors. Each laser beam is split into an illuminating beam and a reference beam which are co-polarized for each wavelength. The two laser beams illuminate the object under interest with illuminating angles $\theta_{x y}$ and $\theta_{z}$. The smooth and plane reference waves are produced through the two spatial filters (SF1 and SF2). Thus each reference wave is the spatial carrier of each hologram.

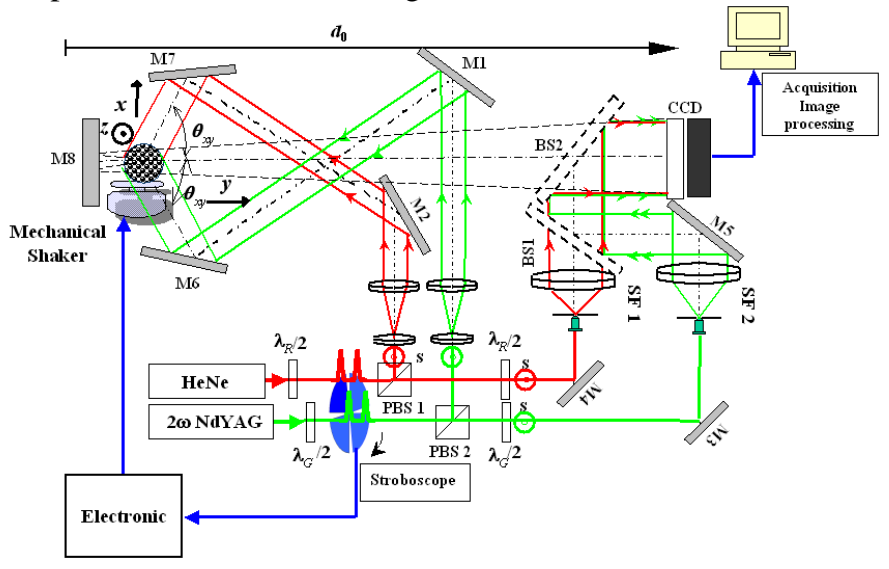

Fig. 1. Two-color experimental set-up (M: Mirrors, BS: beam splitter; SF: spatial filter; PV: mechanical shaker)

Since the monochrome sensor is not able to record the two colors simultaneously at each pixel, the spatial frequencies of the reference waves $(R$ and $G)$ are adjusted so that the two-color holograms are spatially multiplexed in the field of view. Thus, the off-line holographic recording is carried out using the two spatial filters in which each collimating lens is displaced out of the afocal axis by means of two micrometric transducers (not represented in Fig. 1). Light pulses are produced simultaneously using a four sector mechanical shopper. An electronics system is used to synchronize the shopper and the excitation signal put into a mechanical shaker which makes the object vibrating at a controlled amplitude and frequency. As indicated in the previous section, the object under interest is placed at $d_{0}=1400 \mathrm{~mm}$ from the recording area and the illuminated area is $53 \mathrm{~mm}$ in diameter. The digital two-color holograms are reconstructed with $\left\{K_{G}, L_{G}\right\}=\{2000,2000\}$, $d_{G}=d_{0}=-1400 \mathrm{~mm}$ and $\lambda_{G}=532 \mathrm{~nm}$ for the $G$ one, then the $R$ one is reconstructed with $\left\{K_{R}, L_{R}\right\}=\{2378,2378\}, d_{R}=-1399.4424 \mathrm{~mm}$ and $\lambda_{R}=632.8 \mathrm{~nm}$. The sampling pitches of the two images are the same at a precision of $10^{-4} \mathrm{~mm}$. Useful zones in $R$ and $G$ reconstructions can be extracted and pixel-to-pixel superimposed.

Consider that the vibrating object produces a $3 \mathrm{D}$ displacement vector according to:

$$
\mathbf{U}(t)=u_{x} \sin \left(\omega_{0} t+\varphi_{x}\right) \mathbf{i}+u_{y} \sin \left(\omega_{0} t+\varphi_{y}\right) \mathbf{j}+u_{z} \sin \left(\omega_{0} t+\varphi_{z}\right) \mathbf{k}
$$


where $\left\{u_{x}, u_{y}, u_{z}\right\}$ are the maximum amplitudes at pulsation $\omega_{0}=2 \pi f_{0},\left\{\varphi_{x}, \varphi_{y}, \varphi_{z}\right\}$ are the mechanical vibrating phase along $\{x, y, z\}$. The phase changes measured along each color are given by:

$$
\Delta \varphi_{R}=\frac{2 \pi}{\lambda_{R}}\left[\sin \theta_{x y} u_{x}-\cos \theta_{x y} u_{y}+\left(1+\cos \theta_{z}\right) u_{z}\right]
$$

and

$$
\Delta \varphi_{G}=\frac{2 \pi}{\lambda_{G}}\left[-\sin \theta_{x y} u_{x}-\cos \theta_{x y} u_{y}+\left(1+\cos \theta_{z}\right) u_{z}\right]
$$

These equation show that the $x$ movement can be extracted easily and that, with no further processing, one can only obtain a mixing between $z$ and $y$ movement. Designing by $u_{y z}=-\cos \theta_{x y} u_{y}+\left(1+\cos \theta_{z}\right) u_{z}$ the mixing of $y$ and $z$ components, then the difference and sum of the phase changes, multiplied by their respective wavelength allows respectively extraction of the $x$ component and a mixing between $y$ and $z$ components, according to:

$$
\begin{aligned}
& u_{x}=\frac{\lambda_{R} \Delta \varphi_{R}-\lambda_{G} \Delta \varphi_{G}}{4 \pi \sin \theta_{x y}} \\
& u_{y z}=\frac{\lambda_{R} \Delta \varphi_{R}+\lambda_{G} \Delta \varphi_{G}}{4 \pi}
\end{aligned}
$$

Since the $z$ component represents the global out-of-plane vibration mode, one can compute an approximation $\tilde{u}_{y z}$ of $u_{y z}$ by fitting with $n$ smooth Zernike polynomials. Thus, approximation is $\tilde{u}_{y z}=\sum_{i=1}^{n}\left\langle P_{i}, u_{y z}\right\rangle P_{i}$ in with $P_{i}$ are the Zernike polynomial of rank $i$ and $\left\langle P_{i}, u_{y z}\right\rangle$ is the scalar product between $P_{i}$ and $u_{y z}$. So components $u_{y}$ and $u_{z}$ can be now extracted according to:

$$
u_{z}=\frac{\tilde{u}_{y z}}{1+\cos \theta_{z}} \quad u_{y}=-\frac{u_{y z}-\tilde{u}_{y z}}{\cos \theta_{z}}
$$

Since the size of the reconstructed holograms are controlled along each wavelength, the two-color holograms can be pixel to pixel superimposed thus allowing the 3D motion computation according to Eq. 3-7.

For the estimation of the $3 \mathrm{D}$ motion at a given frequency, it is necessary to compute phase differences between stroboscopic recordings and to estimate amplitude and phase of the vibration using a dedicated algorithm. The measurement principle leads to a 3-frame phase shifting algorithms, described in [9], which allows the determination of 3D amplitude and phase of the movement.

\section{Experimental results}

The unconsolidated material under interest is composed of granular materials made of glass $0.15 \mathrm{~mm}$ in diameter. They are excited in the frequency range $f_{0}=[400 \mathrm{~Hz}-3000 \mathrm{~Hz}]$ by using the mechanical shaker. The studied field is a circular wobble plate that is $30 \mathrm{~mm}$ in height and $53 \mathrm{~mm}$ in diameter, and filled with granular. The wobble plate is filled at $98 \%$, thus the studied zone is constituted approximately of 300 grains.

From the 3D estimation can be computed the mean quadratic velocity of the medium, given by: 


$$
\left\langle|v|^{2}\right\rangle=\frac{2 \pi^{2} f_{0}^{2}}{S} \iint_{S}\left(u_{x}^{2}(x, y)+u_{y}^{2}(x, y)+u_{z}^{2}(x, y)\right) d_{x} d_{y}
$$

with $S$ being the surface of the investigated zone. Fig. 2 shows the mean quadratic velocity of the media computed form the experimental results.

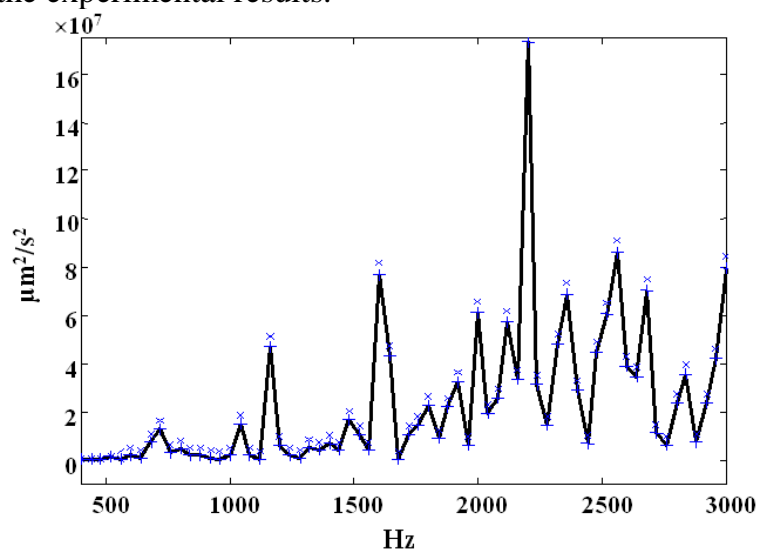

Fig. 2. Mean quadratic velocity in the range $400 \mathrm{~Hz}-3000 \mathrm{~Hz}\left(\mu \mathrm{m}^{2} / \mathrm{s}^{2}\right)$

Fig. 3 shows 3D results at $2760 \mathrm{~Hz}$ proving that the determination of individual vibration amplitudes and phases are made possible by using the Zernike polynomial fitting.

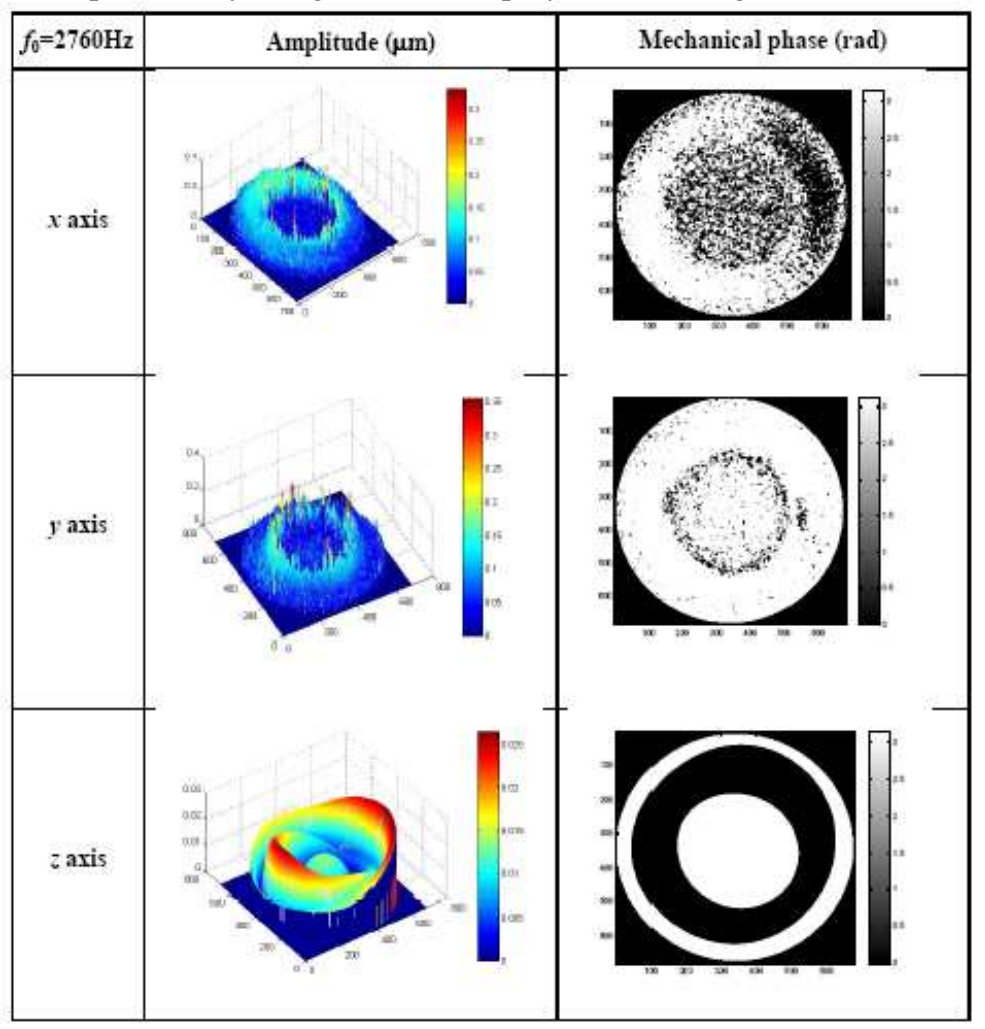

Fig. 3. 3D vibration amplitude and phase at $2760 \mathrm{~Hz}$ 
Fig. 4 shows the 2D in-plane displacement vector at $2760 \mathrm{~Hz}$ which emphasizes the existence of inplane swirling modes.

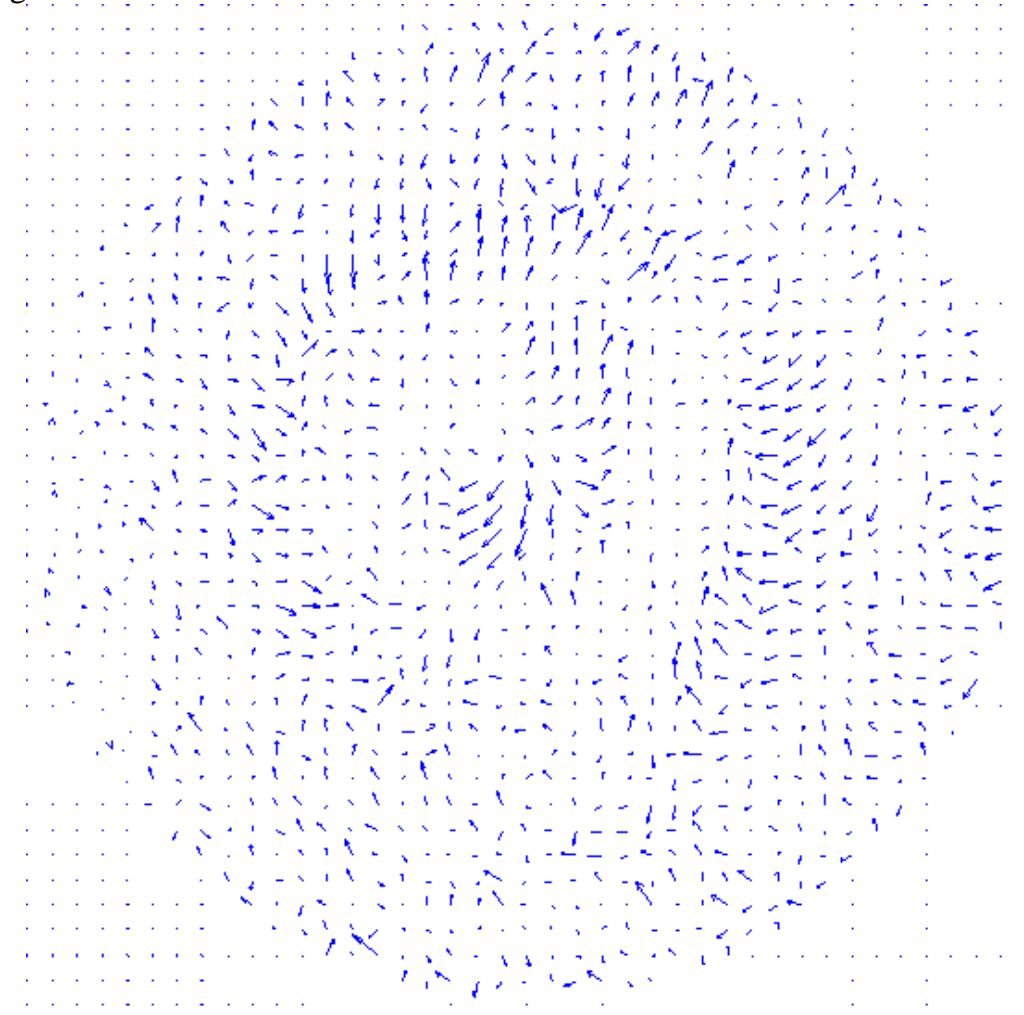

Fig. 4. 2D in-plane vibration vector at $2760 \mathrm{~Hz}$

These experimental results constitute a first attempt to investigate the $3 \mathrm{D}$ movement of unconsolidated materials.

\section{Conclusion}

The paper has presented a first investigation of 3D vibration of granular media using a two-color digital holographic interferometer. The proposed method is based on a spatial-color-multiplexing scheme in which holographic reconstruction is performed using adapted wavelength zero-padding and reconstructing distance. The choice of the reconstruction parameters has been discussed and they guaranty the invariance of the size of the reconstructed object and a constant sampling pixel pitch. The first results on full field analysis of granular media were presented in the frequency band $400 \mathrm{~Hz}-3000 \mathrm{~Hz}$. Complementary experiments are in progress in our lab, but the complex character of medium elasticity does not allow any conclusion to be drawn. However, to the best of our knowledge, results presented in this paper constitute a first attempt to visualize in plane vibration modes in granular medium.

\section{References}

1. U. Schnars, W. Jüptner, App. Opt. 33, 179 (1994).

2. T. Zhang, I. Yamaguchi, Opt. Lett. 23, 1221 (1998).

3. S. Seebacher, W. Osten, T. Baumbach, W. Juptner, Opt. \& Las. Eng. 36, 103 (2001). 
4. P. Picart, J. Leval, M. Grill, J.-P. Boileau, J.C. Pascal, J.-M. Breteau, B. Gautier, S. Gillet, Opt. Expr. 13, 8882 (2005).

5. J.P. Wittmer, A. Tanguy, J.-L. Barrat, L. Lewis, Europhys. Lett. 57, 423 (2002).

6. P. Picart, D. Mounier, J.M. Desse, Opt. Lett. 33, 276-278 (2008).

7. P. Ferraro, S. De Nicola, G. Coppola, A. Finizio, D. Alfieri, G. Pierattini, Opt. Lett. 29, 854 (2004).

8. P. Picart, J. Leval, JOSA A 25, 1744-1761 (2008).

9. J. Leval, P. Picart, J.P. Boileau, J.C. Pascal, Appl. Opt. 44, 5763-5771 (2005). 